\title{
L'initiative récifs coralliens pour le Pacifique (programme CRISP) : bilan des connaissances acquises
}

Bernard Salvat et Clive Wilkinson

\section{(2) OpenEdition \\ Journals}

Édition électronique

URL : http://journals.openedition.org/jso/4792

DOI : $10.4000 /$ jso.4792

ISSN : $1760-7256$

Éditeur

Société des océanistes

Édition imprimée

Date de publication : 15 décembre 2008

Pagination : 75-94

ISBN : 978-2-85430-012-3

ISSN : 0300-953x

Référence électronique

Bernard Salvat et Clive Wilkinson, «L'initiative récifs coralliens pour le Pacifique (programme crisP) bilan des connaissances acquises », Journal de la Société des Océanistes [En ligne], 126-127| Année 2008, mis en ligne le 15 décembre 2011, consulté le 18 juin 2020. URL : http:// journals.openedition.org/jso/4792 ; DOI : https://doi.org/10.4000/jso.4792 


\title{
L'initiative récifs coralliens pour le Pacifique (programme CRISP) : Bilan des connaissances acquises
}

\author{
par
}

\author{
Bernard SALVAT* et Clive WILKINSON**
}

\section{RÉSUMÉ}

Le présent rapport est un compte-rendu des résultats du Programme CRISP quant à l'avancement des connaissances scientifiques en 2006 et 2007 concernant la structure et le fonctionnement des récifs coralliens. Il relève les connaissances acquises au plan général des récifs coralliens et celles concernant les récifs du Pacifique. Il ne s'agit pas d'un rapport sur les activités du CRISP mais de leurs apports à la connaissance fondamentale des récifs. Dans ce cadre, les documents pris en compte dans le présent rapport sont les articles scientifiques publiés dans des périodiques, les communications lors de symposiums, congrès, et colloques. Un certain nombre d'articles sous presse (manuscrits acceptés pour publication dans des revues) ont été pris en compte. Il en est de même de certaines données figurant dans les rapports édités par le CRISP et qui sont sur le site du programme, dans la mesure où certains contenus relèvent de ce que nous indiquons dans le premier paragraphe de ce préambule. Les listes des articles, des communications de symposiums, et des rapports sont données en annexe. Après une introduction générale sur le contexte dans lequel a été élaboré le programme CRISP ( ses préoccupations récentes quant à la préservation des récifs coralliens au plan international et national ; un aperçu de l'état de santé des récifs coralliens dans le Pacifique sud, comparativement au reste du monde; la genèse et la mise en auvre du programme), nous présentons le rapport sensu stricto sur les progrès des connaissances scientifiques sur les récifs coralliens acquis lors du déroulement du programme en fin 2007. Puis, nous dressons un bilan quantitatif de la diffusion des connaissances en référence aux périodiques de publications et à la participation des partenaires du CRISP à des symposiums, colloques et congrès.

Mots-CLÉS : récifs coralliens, lagons, côtier, écologie, gestion, Pacifique

\section{ABSTRACT}

This report presents the results obtained within the framework of the CRISP programme in terms of progressing knowledge on the structure and functioning of coral reefs for the years 2006 and 2007. It gathers newly achieved understanding of coral reefs on a general level and at the scale of the Pacific. It is not a report on CRISP activities per se but a report on its contributions to fundamental knowledge on coral reefs. Within this context, the documents considered are scientific articles published into periodicals, symposia, and presentations at conferences and colloquia. A few articles, although still in press (text approved for publication in magazines) have also been considered. Similarly, data extracted from reports published by CRISP and displayed on the programme website, have been selected whenever their content matches the target presented on the first paragraph of this preamble. The Articles, Symposia presentations, and Reports are listed in the appendices.

This document consists of three sections. First, a general presentation of the context within which the CRISP programme was elaborated. This presents the recent and actual concerns (since 1980) on coral reefs conservation at national and international levels. It gives a general picture of coral reefs health in the South Pacific, by comparison with the rest of the world. It relates the initiation and implementation of the CRISP programme. Second, reports stricto sensu, on coral reefs scientific knowledge progress gained during the programme's evolution up to late 2007. Third is a quantitative assessment of knowledge dissemination, including periodical publications and CRISP partners' participation in symposia, lectures and conferences.

KEYwORDs: coral reefs, lagoons, coastal, ecology, mangement, Pacific 


\section{Le contexte du programme CRISP}

Introduction sur l'intérêt récent porté aux récifs coralliens au plan international et national

La dégradation des écosystèmes coralliens dans les trois océans a été soulignée par la communauté scientifique dès les années 1980. Les congrès internationaux sur les récifs coralliens ont alors comporté des sessions sur ce sujet. La pollution par les activités humaines, le développement de l'urbanisation et du tourisme, les dégradations directes du milieu par un développement incontrôlé des zones côtières, la surexploitation des ressources récifales en raison d'une pression démographique trop forte, la poursuite de techniques de pêche dommageables pour le milieu corallien, sont des sujets qui furent abordés lors des congrès de Manille (1981), de Tahiti (1986) et des suivants. La nécessité d'actions visant à réduire les interventions humaines néfastes aux récifs coralliens s'est imposée de même que l'urgence de développer les réserves marines (Salvat, 1983, 1987).

La préoccupation des changements climatiques émergea en donnant un coup de projecteur sur les récifs coralliens à la fin des années 1980. Des groupes de travail ont été constitués par la COI-UNESCO (Commission océanographique intergouvernementale), avec le PNUE (Programme des Nations unies pour l'environnement), avec l'omm (Organisation météorologique mondiale) puis l'UICN (Union internationale pour la conservation de la nature, maintenant Union mondiale pour la nature) pour débattre et donner leurs conclusions sur les effets des changements climatiques sur les récifs coralliens. Deux prédictions étaient particulièrement considérées : l'élévation de la température et celle du niveau des océans (IOC et al., 1991, 1992, 1993, Wilkinson and Buddemeier, 1994). La première était illustrée par les phénomènes de blanchissements établis depuis les années 1960 (Goreau, 1964) mais qui se multipliaient dans les zones coralliennes des trois océans. Une très faible élévation de la température estivale des eaux provoque une rupture de l'association des coraux avec les algues symbiotiques qu'ils hébergent dans leurs tissus; ces derniers alors translucides laissent apparaître le squelette calcaire blanc $\mathrm{du}$ corail, d'où le terme de blanchissement. Ce phénomène provoquait parfois chez les coraux des mortalités massives dont la fréquence, l'intensité et la répartition géographique avaient considérablement augmenté au cours des décennies 1970 et 1980 . La seconde prédiction, l'élévation du niveau des océans, était très préoccupante pour les populations humaines, mais non pour les récifs coralliens qui gagneraient quant à eux en surface et en importance, comme l'ont conclu les rapports des groupes de travail à l'époque. Conjointement à l'intérêt porté sur les récifs coralliens comme sentinelle des changements climatiques, la nécessité d'un suivi de l'état de santé des récifs coralliens à l'échelle mondiale se faisait jour (Salvat, 1991) et un groupe de travail international recommandait en 1992 (IOC et al., 1992) la constitution de ce qui allait devenir en 1995 le réseau mondial de surveillance du milieu corallien, le GCRMn (Global Coral Reef Monitoring Network), qui publia son premier bilan mondial en 1998 (Wilkinson, 1998).

C'est dans le contexte de cette prise de conscience croissante de la détérioration des récifs coralliens, de la nécessité de les préserver, de l'inquiétude des changements climatiques et du besoin d'établir régulièrement leur état de santé à l'échelle planétaire, que les récifs coralliens, haut lieu de la biodiversité, font l'objet d'une attention particulière de la part des gouvernements en 1994. Le Département d'État des États-Unis lance une initiative internationale en faveur des récifs coralliens, l'ICRI (International Coral Reef Initiative), en dehors du système des Nations unies, à laquelle se joignent plusieurs pays : Australie, France, Royaume-Uni, Japon, Jamaïque, Philippines, Suède et diverses agences internationales (Banque mondiale, PNUE) et organisations non gouvernementales (UICN) auxquels se sont associés par la suite de nombreux autres partenaires. L'ICRI est une structure informelle qui vise à la concertation des actions en faveur des récifs coralliens, préservation et gestion durable. Le secrétariat de l'ICRI, dont les réunions sont annuelles, est assuré par un pays ou un couple de pays qui est renouvelé tous les deux ans: USA, Australie, France (1999-2000), SuèdePhilippines, United Kingdom-Seychelles, JaponPalau, USA-Mexique (2007-2009). Un appel à action et un plan d'action, établis en 1995, ont été adoptés par plus de quatre-vingts pays sur les quelques cents pays, presque tous en voie de développement, qui possèdent des récifs coralliens sur leurs côtes. L'ICRI a réussi à faire en sorte que les récifs coralliens soient considérés dans les ordres du jour, les préoccupations et les recommandations des gouvernements et des organisations internationales. Les conventions internationales comme celles sur la diversité biologique (CBD) ou le développement durable (CDD) traitent des récifs coralliens, de même que des rapports plus larges sur la pauvreté (Whittingham et al., 2003) ou les changements clima- 
tiques (Groupe d'experts internationaux sur le changement climatique, IPCC).

Assurant le secrétariat de l'ICRI en 1999 et 2000, la France a lancé l'IFRECOR (Initiative française sur les récifs coralliens). Sous l'égide du ministère de l'Écologie et du Développement durable et du ministère de l'Outre-mer, a été créé un comité national comportant des parlementaires et des représentants des administrations concernées, des représentants des comités locaux organisés dans chaque collectivité outre-mer avec récifs coralliens (Martinique, Guadeloupe, Réunion, Mayotte, Îles Éparses, NouvelleCalédonie, Wallis-et-Futuna et Polynésie française), d'associations scientifiques et techniques et de socio professionnels. Un plan d'action national a été établi pour 2000-2005 et les résultats analysés pour la formulation d'une seconde phase 2006-2010. La sensibilisation et la prise en compte des récifs coralliens dans les politiques publiques, notamment liées à la sauvegarde de la biodiversité (SNB, Stratégie nationale de la biodiversité) et à la nécessité d'un développement durable (SNDD), sont à l'actif de l'IFRECOR. Les budgets IFRECOR délivrés par les deux ministères de tutelle permettent aux comités locaux de participer à des actions nationales et de faire réaliser ou de participer à des travaux dans leurs contextes propres, grâce à une concertation avec les organismes de recherche et les besoins des services compétents en matière de gestion environnementale.

Autour de réunions interministérielles à Paris en 2002, et dans la perspective du Sommet France-Océanie qui s'est réuni à Papeete en juillet 2003 sous la présidence de Jacques Chirac, s'est organisée une réflexion sur la préparation d'un programme français sur les récifs coralliens des états du Pacifique sud, voisins de la Nouvelle-Calédonie, de Wallis-et-Futuna et de la Polynésie française. Un tel programme sur la protection et la gestion des récifs coralliens avait aussi pour ambition de développer la coopération entre partenaires français et anglo-saxons de la zone. C'est dans ce contexte général de développement de l'ICRI, de l'IFRECOR, de l'intérêt croissant porté aux récifs coralliens et aux populations qui en vivent, qu'était lancée en 2004 l'initiative pour la protection et la gestion durable des récifs coralliens dans le Pacifique Sud, programme CRISP pour Coral Reef Initiative for the South Pacific, répondant par ailleurs à une volonté politique de voir les collectivités outre-mer du Pacifique mieux insérées régionalement. Depuis, une extension du programme s'est faite dans l'hémisphère Nord (notamment à Palau) mais l'acronyme CRISP a été conservé alors que l'initiative ne se restreint plus au Pacifique Sud.

\section{L'état de santé des récifs coralliens du Pacifique sud}

Le dernier bilan de l'état de santé des récifs dans le monde établi par le GCRMN date de 2004 (Wilkinson, 2004). Parmi les dix-sept régions des trois océans dans lesquelles sont établis ces bilans, celles qui nous intéressent dans le cadre du programme CRISP sont les régions du «sud-ouest Pacifique» (Lowell et al., 2004) et du « centre et sud-est Pacifique » (Vieux et al., 2004), avec en complément la Papouasie Nouvelle-Guinée.

Il y a cent ans, les récifs du Pacifique Sud étaient en excellente condition avec une faible pression humaine. L'économie de subsistance était la règle avec des pratiques de pêche ancestrales. On pouvait noter toutefois quelques méthodes destructives de pêche (poisons) et l'exploitation intensive de certaines ressources pour commercialisation vers l'Europe ou l'Asie comme la nacre à partir des atolls polynésiens et le trépang (holothuries) à partir des lagons mélanésiens.

Les récifs du Pacifique Sud sont actuellement les moins dégradés du monde. Si les récifs du sud et du sud-est asiatique sont détruits à près de $40 \%$, il n'en est rien du Pacifique Sud où une telle situation n'a concerné que 2 à $3 \%$ de leur surface totale. Les récifs menacés ne représentent que 2 à $3 \%$ dans les eaux polynésiennes alors que la proportion dépasse les $30 \%$ sur les côtes asiatiques (Wilkinson, 2004). Les activités humaines dans le Pacifique Sud concourent néanmoins à une certaine dégradation surtout dans les zones urbanisées. Les phénomènes naturels (cyclones, blanchissements et mortalité des coraux...) agissent sur l'ensemble des récifs de la région avec une préoccupation croissante en raison des prédictions des changements climatiques.

Les récifs coralliens à proximité immédiate des zones urbaines des États du Pacifique Sud ont été détruits par remblaiement pour la construction d'infrastructures portuaires et aéroportuaires, conjointement à un développement côtier souvent incontrôlé. Les routes en périphérie des îles ont rapidement aligné les contours sinueux des rivages avec remblai des récifs frangeants. Les constructions hôtelières et les ports de plaisance se font toujours au détriment des récifs frangeants, tout comme les extractions de matériaux coralliens pour les besoins du développement. L'activité minière est la cause d'une éro- 
sion importante extrêmement dommageable pour les récifs. Une mauvaise gestion des terres par défrichement pour l'urbanisation ou l'agriculture a entraîné des apports sédimentaires et chimiques néfastes aux récifs coralliens, une des sources de pollution la plus générale et la plus importante pour les récifs coralliens dans le monde entier. En zone urbaine, cette pollution s'accompagne de celle des eaux usées car les réseaux et stations de traitement des eaux sont exceptionnels. L'enrichissement des eaux lagonaires en nitrates et phosphates a favorisé le développement des algues au détriment des coraux dans le contexte général de dégradation des zones très habitées. Sur un plan plus général, la pression démographique est de plus en plus forte et surtout de plus en plus concentrée géographiquement et la surexploitation des ressources dans certains secteurs est alarmante.

En dehors des zones urbanisées, les récifs coralliens du Pacifique Sud sont relativement bien protégés par leur isolement naturel. Cet isolement est à double échelle. Les récifs coralliens du Pacifique Sud sont très éloignés des masses continentales et des rejets et dégradations dus aux activités humaines qui s'y déroulent avec une importante pression démographique. À plus petite échelle, les récifs du Pacifique Sud sont des récifs d'îles océaniques très souvent de petite taille à l'exception de Fiji et de la NouvelleCalédonie. Les formations coralliennes sont isolées les unes des autres, mais il est important de noter que leur connectivité est de plus en plus démontrée du fait de la reproduction par larves à phase océanique qui caractérise la quasi-totalité des organismes des récifs.

Les événements naturels catastrophiques sont sans conteste ceux qui provoquent le plus de dommage aux récifs coralliens du Pacifique Sud. Les cyclones peuvent détruire complètement un couvert corallien de pente externe d'un versant d'une île haute ou d'un atoll, comme cela a été constaté en Polynésie française sur l'atoll de Tikehau et Takapoto (Harmelin, Vivien et Laboute, 1983; Laboute 1985). Grâce aux réseaux de surveillance de l'état de santé des récifs, mis en place avec des observations annuelles qui portent bientôt sur plus de deux décennies, on sait maintenant qu'en dehors de toute autre perturbation majeure, le récif mettra une douzaine d'années à se reconstituer. Les phénomènes de blanchissement des coraux peuvent occasionner des mortalités sur de vastes étendues. Enfin, les explosions démographiques de l'étoile de mer épineuse (Acanthaster planci), prédatrice des coraux, mettent à mal des écosystèmes récifaux entiers compte tenu de la concen- tration de cet échinoderme qui se nourrit des parties molles des coraux laissant un récif mort derrière ses hordes.

Ces événements sont naturels et les récifs devraient s'en accommoder, ce qu'il nous faut nuancer. En effet, les récifs sont fragilisés par les conséquences des activités humaines qui perdurent (surtout dans les zones urbaines); ils récupèrent moins bien après les destructions naturelles. La synergie qui s'établit entre ces phénomènes est dommageable pour les récifs. Par ailleurs, les événements naturels destructeurs semblent avoir augmenté en nombre, en étendue et en intensité au cours des dernières décennies et les prédictions des changements climatiques sont préoccupantes.

Les catastrophes naturelles majeures au cours des dernières décennies dans le Pacifique Sud ont été des cyclones en 2003-2004 en NouvelleCalédonie, aux Samoa, aux îles Salomon et au Vanuatu. Pour la Polynésie française, il faut remonter au phénomène El Nino de 1982-1983, pour noter plusieurs cyclones dévastateurs dans les Tuamotu et les îles de la Société. On note aussi des phénomènes importants de blanchissement en 2000-2002 dans l'ensemble du Pacifique Sud avec des mortalités allant jusqu'à $80 \%$ des coraux dans certains secteurs des Fiji. Enfin, les explosions démographiques de l'étoile de mer épineuse qui ont endommagé l'ensemble des récifs coralliens des océans Indien et Pacifique dans les années 1980, existent toujours sporadiquement. Il semble qu'une nouvelle explosion de cette espèce se dessine actuellement dans les îles de la Société.

Le constat est donc établi que les récifs coralliens du Pacifique Sud sont les moins dégradés du monde et que leur devenir est moins alarmant qu'ailleurs. Cependant, la pression démographique, le développement en général y compris le tourisme et la disparition de pratiques ancestrales de conservation de la ressource, sont autant de facteurs qui aggravent la situation des récifs coralliens. Une politique de préservation de ce bien naturel, assise de la culture des peuples océaniens, doit être mise en œuvre en tenant compte des options de développement durable pour le bien être des populations. Les prédictions des changements climatiques sont au centre des préoccupations. Au-delà de la montée du niveau des océans qui affectera négativement les populations mais sans dommage pour les récifs coralliens (ceux-ci étant un facteur de protection du littoral et donc des implantations humaines), il faut mentionner le renforcement annoncé des cyclones (géographiquement et en fréquence), l'élévation de la température des océans avec les 
conséquences des blanchissements et des mortalités coralliennes, sans compter le problème de l'acidification des eaux océaniques qui peut entraîner une perturbation notable chez les organismes calcifiants.

\section{L'initiative récifs coralliens Pacifique CRISP}

L'initiative pour la protection et la gestion durable des récifs coralliens dans le Pacifique Sud est née d'une volonté politique française de contribuer au développement des états insulaires de la région en favorisant l'intégration régionale au sens de l'insertion des collectivités territoriales françaises d'outre-mer dans leur environnement et de la coopération avec les « grands voisins »: Australie, Nouvelle-Zélande, Japon et États-Unis. Elle a été préparée par l'Agence française de développement (AFD) dans un cadre interministériel à partir de l'année 2002, en étroite concertation avec le Secrétariat permanent pour le Pacifique (Premier ministre). Un appel à propositions a été lancé en juin 2003 et tous les répondeurs à l'appel ont été intégrés à l'architecture du programme qui a été établie en juin 2004, pour une validation en juillet par les instances de l'AFD et du Fonds français pour l'environnement mondial (FFEM). Ce programme a été labellisé sous l'acronyme de CRISP à partir de septembre 2004, et lancé officiellement par le ministre français de l'Écologie et du Développement durable, à l'occasion de la Conférence annuelle du PROE (Programme régional océanien pour l'environnement) et de la réunion des ministres de l'Environnement des pays membres du PROE qui se sont tenues à Papeete ce même mois.

Dans ce dessein, l'initiative développe une approche spécifique qui vise à :

- associer activités de réseau et projets de terrain ;

- articuler recherche, aménagement et développement et formation ;

- combiner les apports de disciplines scientifiques diverses, incluant la biologie, l'écologie, l'économie, la sociologie, le droit et les sciences humaines ;

- intervenir sur l'ensemble des thèmes - terrestres et marins - intéressant les récifs (y compris l'assainissement et la gestion des bassins versants) ;

- ne pas créer de structure nouvelle mais apporter des ressources financières à des partenaires déjà opérationnels et souhaitant développer leurs activités dans un esprit de coopération régionale. C'est la raison pour laquelle l'initia- tive a été préparée sur la base d'un appel à propositions auprès de l'ensemble des institutions et réseaux.

Cette approche se décline sur une série d'objectifs thématiques qui sont :

1 : une meilleure connaissance de la biodiversité, de l'état et du fonctionnement des écosystèmes coralliens

$2:$ la réalisation d'opérations de protection et de gestion des écosystèmes coralliens à une échelle significative

3: la valorisation du potentiel économique reposant sur les valeurs d'usage et la biodiversité des écosystèmes coralliens

4 : la diffusion de l'information et des savoirs ; renforcement des capacités et animation des réseaux locaux, nationaux et internationaux.

Le dispositif d'intervention du CRISP se structure en trois composantes majeures :

\section{$1:$ Gestion côtière intégrée}

- 1A1: Planification de la conservation de la biodiversité marine

- 1A2: Aires marines protégées

- 1A3 : Renforcement institutionnel et mise en réseau

- 1A4 : Gestion intégrée des zones côtières récifales et des bassins versants

2 : Développement des écosystèmes coralliens

- 2A : Connaissance, valorisation et gestion des écosystèmes coralliens

- 2B : Restauration récifale

- 2C : Valorisation des substances actives marines

- 2D : Mise en place d'une base de données régionale (Reefbase Pacifique)

3 : Coordination et valorisation du programme

- 3A : Capitalisation, valorisation et vulgarisation des acquis du programme CRISP

- 3B : Coordination, promotion et développement du programme CRISP

Initialement réservé aux PEIP (petits États insulaires du Pacifique) seuls éligibles à une subvention de l'AFD ou du FFEM, le financement du CRISP a pu s'étendre aux collectivités territoriales françaises outre-mer du Pacifique grâce à l'abondement de ce financement initial français par des contributions financières de partenaires tels que CI (Conservation International), le WwF (World Wildlife Fund for Nature) ou l'UNF (United Nations Foundation), sur lesquels ne pesaient pas 
les mêmes contraintes d'éligibilité et qui ont ainsi affirmé leur volonté de collaboration au programme. Ces nouvelles dispositions et possibilités d'actions ont été envisagées lors de la participation d'un expert de l'AFD à la réunion des comités IFRECOR à Mayotte en Mai 2004. La Polynésie française a répondu à cet appel à proposition. La Nouvelle-Calédonie et les îles Wallis-et-Futuna ont aussi rejoint la douzaine de PEIP en tant que bénéficiaires du programme.

Un atelier technique de lancement du programme CRISP, financé par le Fonds de coopération économique, sociale et culturelle pour le Pacifique de la République française (MAE), s'est tenu à Nouméa en janvier 2005 et un premier plan d'action a été défini par tous les partenaires réunis. En attente de la signature des conventions de financement de l'AFD et du FFEM avec ces partenaires, ce plan d'action a été adopté pour actions immédiates.

Le programme CRISP était initialement prévu pour trois années, mais la participation de partenaires internationaux (CI, UNF, WWF) a nécessité la signature de conventions qui n'ont pu être réglées que tardivement avec la mise en place des financements correspondants. Il a été décidé en 2007 que le programme serait prorogé jusqu'en fin 2009 avec des crédits complémentaires de l'AFD et du FFEM pour renforcer les actions prometteuses et tenir compte de nouvelles problématiques apparues depuis la formulation du programme en 2004. Le programme est géré par la cellule de coordination implantée au secrétariat de la Communauté du Pacifique Sud (CPS) à Nouméa.

\section{Les progrès des connaissances scientifiques}

Nous abordons ici les progrès des connaissances scientifiques sur les récifs coralliens au cours des deux premières années de plein exercice du programme CRISP (2006 et 2007) par analyse des publications dans des périodiques ainsi que des communications lors de colloques et de quelques rapports à diffusion restreinte lorsque ceux-ci apportent des données nouvelles. Cette analyse est faite selon les quatre axes suivants :

1 : les progrès de la connaissance sur la biodiversité des écosystèmes coralliens en termes d'habitats et de richesse en espèces qui seront des éléments et des arguments pour une meilleure gestion du patrimoine naturel ;

2 : les progrès de la connaissance sur la structure et le fonctionnement des écosystèmes coralliens qui seront des éléments et des arguments pour la mise au point d'outils de gestion adaptés et performants ;

3 : les progrès de la connaissance sur de nouvelles ressources et leur potentiel d'exploitation économique ;

4 : les progrès dans la maîtrise de nouveaux outils de surveillance de l'état des habitats et des ressources et de leur gestion.

Trois tableaux en annexes donnent la liste des éléments de notre analyse selon trois catégories : « $\operatorname{articles}(\mathrm{A}) »$, « communications symposiums $(\mathrm{S}) »$ et « rapports $(\mathrm{R}) »$. L'axe dans lequel nous allons traiter chaque élément est indiqué dans les tableaux, mais le choix n'est pas toujours incontestable. Les rapports et les communications symposiums qui décrivent le programme CRISP et ses objectifs ne sont pas pris en compte dans notre analyse car ils ne donnent aucun résultat de recherche.

Axe 1 : les progrès de la connaissance sur la biodiversité des écosystèmes coralliens en termes d'habitats et de richesse en espèces

Plusieurs groupes taxonomiques ont fait l'objet de recherches poussées amenant la description d'espèces nouvelles (ou de stades de vie inconnus d'espèces), l'établissement d'inventaires dans certaines régions ou des travaux biogéographiques.

Les poissons sont ceux qui ont été le mieux étudiés par la description jusqu'alors inconnue de leurs stades larvaires. Cette recherche amont était indispensable au développement de la filière d'élevage des larves collectées sur les récifs pour différents objectifs économiques et écologiques. Pour 140 espèces de poissons des récifs coralliens, les stades larvaires, photographiés et caractérisés morphologiquement, ont été décrits dans plusieurs documents largement diffusés (rapport 3 ). Un guide d'identification de stades jeunes de 70 espèces de poissons de Wallis et du Pacifique central a également été édité (rapport 2). Ces nouvelles données ne sont accessibles que dans des publications nécessairement en couleur; elles peuvent difficilement faire l'objet d'articles dans des périodiques scientifiques sauf pour indiquer le progrès des connaissances à ce sujet et indiquer où elles sont disponibles avant qu'elles ne se trouvent publiées dans des ouvrages de référence spécialisés. Ces descriptions ouvrent tout un volet de recherche sur les stades larvaires et juvéniles des poissons coralliens.

Les coraux scléractiniaires ont fait l'objet d'un inventaire en Nouvelle-Calédonie à partir de collections rassemblées depuis plusieurs années à l'IRD et de prospections nouvelles sur le terrain. 
Les collections de coraux à l'IRD étaient la résultante de récoltes depuis plusieurs décennies; elles méritaient d'être revues et valorisées pour une conservation durable (rapport 22). Au total 215 espèces de 69 genres y ont été identifiées alors que certains taxa restent d'identification problématique (Acropora et Montipora). Des prospections terrain dans la région du Diahot au nord de la Nouvelle-Calédonie (WWF, province Nord, CRISP dans le cadre de l'analyse écorégionale de Nouvelle-Calédonie) ont permis d'identifier 216 espèces de scléractiniaires sur 27 stations prospectées, dont 3 qui n'avaient jamais été recensées en Nouvelle-Calédonie (rapport 21). Un rapport de synthèse sur les coraux de Nouvelle-Calédonie fait état de 310 espèces en 74 genres répartis en 17 familles (rapport 24). Ainsi la richesse spécifique en coraux de Nouvelle-Calédonie est elle mieux établie, approchant celle de la Grande barrière de corail australienne, et les collections IRD ont elles été valorisées en vue d'une meilleure conservation. D'autres informations dans le cadre du CRISP ont été établies sur les coraux de NouvelleCalédonie, mais elles concernent leur écologie et seront évoquées plus loin. Ces données sur les coraux ont contribué à étoffer le dossier de demande de classement des récifs calédoniens au patrimoine mondial de l'Humanité de l'Unesco.

Les crustacés ont été l'objet d'inventaire dans les îles Wallis, Futuna et Alofi (rapport 25). La prospection de 31 stations a donné lieu à la récolte et à l'identification de 115 espèces ( 2 stomatopodes, 3 langoustes, 1 cigale, 17 crevettes, 29 anomoures et 63 crabes). C'est le premier inventaire de crustacés de Wallis et Futuna qui n'a fait l'objet que d'un rapport de mission qui prévoit une liste complète qui sera publiée en fin 2008, l'édition d'un guide d'identification d'une centaine d'espèces de crustacés les plus communs du Pacifique et un article exhaustif dans Atoll Research Bulletin. Par ailleurs, un article sur la biogéographie des crustacés (article 1) est à paraître dans un volume spécial de la revue Pacific Science, volume spécial que rend compte d'un séminaire franco-japonais sur la biodiversité qui s'est tenu sous l'égide du CRISP.

Les algues ont également fait l'objet d'inventaires aux îles Salomon et au Vanuatu où ont été dénombrées 284 espèces (272 Rhodophytes, Chlorophytes et Ochrophytes -8 phanérogames marines -4 cyanobactéries) (rapport 23). Deux espèces d'algues rouges nouvelles pour la science ont été décrites (Myriogramme melanesiensis et $M$. heterostroma) (article 2) ainsi qu'un nouveau genre et espèce (Grammephora peyssonnelloides) (article 3). Par ailleurs les espèces associées au genre Turbinaria ont fait l'objet d'une mise au point (article 4) le manuscrit de description d'une autre espèce nouvelle est sous presse ( $\mathrm{Seb}$ denia cerebriformis) alors que 6 autres espèces nouvelles sont en cours de description (Martensia, Rhizophillis, Rhodymenia, Dumontiaceae, Hypoglossum, Dudresmaya) (rapport 23).

Ces récoltes et inventaires n'ont pas fini d'apporter un enrichissement à la connaissance de la biodiversité des récifs coralliens prospectés. Non seulement des inventaires précis ont été réalisés dans des zones parfois encore non prospectées, mais l'effort de recherche qui a déjà donné lieu à la description de nombreuses espèces nouvelles pour la science, va se poursuivre dans les prochaines années.

En terme de biodiversité d'habitats, il s'agit surtout de l'atlas qui a été produit sur les récifs coralliens du sud-est de la Papouasie NouvelleGuinée (rapport 5). Cet atlas fait pendant à celui publié par l'IRD sur les récifs de NouvelleCalédonie. Il s'agit là de réalisations incontournables pour toute étude écologique dans ces régions. Il est opportun de spécifier que dès la mi-2008, un atlas des récifs coralliens pour le Pacifique répondant à la même approche que celle appliquée en Papouasie Nouvelle-Guinée, sera mis à disposition par le programme CRISP sous la forme d'un CD distribué à l'ICRs2008 en Floride (USA) et ensuite proposé gratuitement en ligne sur le portail internet Reefbase Pacific. Il s'agit là d'une source d'information particulièrement précieuse, tant sur le plan quantitatif que qualitatif, pour le monde scientifique et les gestionnaires. Par ailleurs, lors d'un symposium, une communication a été présentée sur la cartographie des habitats des récifs côtiers des îles Fiji (symposium 1).

Il convient enfin de mentionner une contribution à un ouvrage juridique sur le droit de l'environnement, issue en partie de la collaboration d'une juriste au programme CRISP et portant sur la «protection juridique de la biodiversité marine » (article 5).

Axe 2 : les progrès de la connaissance sur la structure et le fonctionnement des écosystèmes coralliens

Plusieurs thèmes de recherches ont été abordés et ont donné lieu à des publications ou à des communications lors de symposiums en complément de rapports qui annoncent de futurs articles dans des périodiques. Nous aborderons successivement les thèmes suivants : connectivité, recrutement et habitats, biologie des poissons, surveillance de l'état de santé des récifs face aux 
perturbations, écologie côtière et restauration des récifs dégradés.

La connectivité entre les récifs avec l'étude de la dispersion des larves a été abordée dans un article de Science: "Local Replenishment of Coral Reef Fish Populations in a Marine Reserve » (article 8). Dans une île aire marine protégée, le devenir des larves d'un poisson sédentaire associé à des anémones (Amphiprion percula) et d'un poisson papillon vagile (Chaetodon vagabundus) est examiné. Environ $60 \%$ des larves issues de parents de l'île recrutent dans l'île, que la durée du stade larvaire de l'espèce soit inférieure à deux semaines ou supérieure à un mois. Cet autorecrutement est important et constitue un éclairage important sur les problèmes de connectivité entre les récifs qui sont abordés à des échelles spatiales très variables et dont on doit tenir compte pour la délimitation des aires marines protégées.

Summary: The scale of larval dispersal of marine organisms is important for the design of networks of marine protected areas. We examined the fate of coral reef fish larvae produced at a small island reserve, using a mass-marking method based on maternal transmission of stable isotopes to offspring. Approximately $60 \%$ of settled juveniles were spawned at the island, for species with both short ( $<2$ weeks) and long ( >1 month) pelagic larval durations. If natal homing of larvae is a common life-history strategy, the appropriate spatial scales for the management and conservation of coral reefs are likely to be much smaller than previously assumed.

Cette recherche fait également l'objet d'une communication dans un symposium (symposium 18). À une échelle plus large, la dispersion des larves de poissons dans l'ensemble de l'indopacifique est incontestable comme le montrent les études génétiques (symposium 19).

Les problèmes du recrutement des larves et juvéniles en relation avec les caractéristiques de l'habitat ont été étudiés pour les poissons et les invertébrés (articles 6 et 7 et symposiums 5, 10, 14). Ces travaux mettent l'accent sur les stimuli sensoriels qui déterminent chez les larves ou les juvéniles un recrutement préférentiel dans les habitats qui leur conviennent. Ces études sont récentes et capitales pour, au-delà de la dispersion des larves et de la connectivité des récifs, expliquer et agir pour un meilleur recrutement des espèces récifales qu'elles soient d'intérêt commercial ou tout simplement pour maintenir la biodiversité.

Les travaux sur la biologie des poissons récifaux ont été l'objet de plusieurs articles et de communications lors de symposiums. Leur nombre s'explique par l'intérêt suscité par les res- sources halieutiques concernant un groupe qui représente une source de protéines importante pour les populations du Pacifique sud. L'étude de la croissance de juvéniles d'un poisson (Pomacentrus molluccensis) dans son habitat (symposium 43 et article 9 à soumettre à Aquatic Bio$\log y)$ montre que les caractéristiques de l'habitat sont importantes pour déterminer l'abondance du peuplement, nouveaux venus et adultes déjà résidents, par opposition à l'hypothèse d'une réduction de la croissance des nouveaux recrutés dus au surpeuplement. Une autre publication est envisagée sur ce thème en considérant plusieurs espèces (Chromis viridis, Lethrinus genivittatus et Siganus fuscescens) (article 10 à soumettre à Oecologia). Ces résultats, comme les précédents, font ressortir l'importance de l'habitat dans le maintien de la biodiversité des populations et de leurs abondances et la nécessité de tenir compte de ces informations lors de la création et des réglementations dans les aires marines protégées. Les traits de vie des poissons récifaux constituent un axe de recherche à développer en vue d'une meilleure connaissance des conditions de maintien de leurs populations dans les récifs (symposium 23). Dans le fonctionnement des récifs, la diversité-richesse spécifique des poissons n'est pas le seul élément à prendre en compte ; il convient de considérer aussi les biomasses des diverses espèces groupées selon leur régime alimentaire (symposium 24). Une dernière communication symposium (symposium 9) traite de la diversité des communautés de poissons sous influences naturelles et anthropiques.

La surveillance de l'état de santé des récifs, dans un cadre général d'évolution des communautés récifales en fonction des perturbations naturelles et anthropiques ou dans le cadre du suivi d'une perturbation majeure particulière, a fait l'objet de plusieurs communications lors de symposiums $(3,12,13,20,21,22)$ et de rapports $(14,15,17,18,19)$. Ces travaux réalisés aux Fiji et en Polynésie française ont un intérêt régional mais contribuent au bilan de santé mondial des récifs coralliens qui est établi par le GCRMN de l'ICRI. En effet, dans ce cadre, la surveillance de l'état de santé des récifs coralliens dans le Pacifique Sud est assurée par deux centres, Fiji et Polynésie française, qui regroupent autour d'eux les pays riverains. Les derniers bilans ont été publiés en 2004 (Status of Coral Reefs of the World) dans l'ouvrage de Wilkinson. Le CRISP aide à la pérennité de cette surveillance depuis 2005 dans certains pays dépendant du réseau centré sur Fiji (Institute of Marine Resources, USP). De même, le CRISP a permis le lancement à plein régime d'un réseau de surveillance Reef 
Check des récifs en Polynésie française conjointement avec Reef Check International, IFRECOR Polynésie et la Fondation d'entreprise tотAL. L'action essentielle du CRISP dans le domaine de la surveillance de l'état de santé des récifs porte sur le financement de coordinateurs des deux réseaux implantés à Fiji (USP) et à Moorea (CRIOBE) et sur la liaison à assurer avec le World Fish Center de Penang (Malaisie) qui gère la base de données ReefBase. Cette base mondialement connue sur les récifs et leur exploitation est la base de référence et de dépôt des données traitées par le GCRMN à l'échelle mondiale. Le CRISP s'est attaché à donner une plus grande cohérence au niveau régional quant à la collecte des données, à leur traitement et à leur disponibilité sur le site ReefBase. Cette coordination et ces résultats porteront leurs fruits lorsque sera établi le prochain bilan de santé des récifs coralliens dans le monde. Il reposera sur des analyses par réseaux, comme ceux centrés sur Fiji et Moorea, et sur une approche régionale à l'échelle du Pacifique Sud. Le GCRMN prévoit d'éditer son rapport en 2008. Indiquons incidemment que sera édité en même temps, à l'initiative de l'IFreCOR, un ouvrage établissant l'état de santé des récifs de toutes les collectivités françaises outre-mer et rendant compte des évolutions récentes. Une communication (symposium 20) mentionne l'inquiétante explosion démographique de l'étoile de mer épineuse (Acanthaster planci) depuis 2006 sur les récifs d'îles hautes en Polynésie. Il est à craindre que cette explosion, comme dans les années 1980, ne s'amplifie et détruise une bonne partie des communautés de scléractinaires alors que celles-ci étaient à leur optimum depuis quelques années. Considérant à Moorea, Polynésie française, des observations sur deux décennies, les modifications des peuplements récifaux (importance relative des différents groupes animaux et végétaux) essentiellement sous influence des facteurs naturels, ont permis d'aborder les questions de résistance et de résilience (symposium 21). Les résultats ont une portée qui dépasse le cadre géographique dans lequel les travaux ont été réalisés en raison de la rareté de séries d'observations sur plusieurs années (plus de vingt ans sur Moorea) concernant l'évolution des récifs.

L'écologie côtière s'est penchée sur les dégradations des récifs coralliens en rapport avec l'environnement des bassins versants correspondants. Les risques de dégradations ont été étudiés en Nouvelle-Calédonie (rapport 6) L'érosion des sols et ses conséquences sur les récifs coralliens étudiés à Fiji, en Nouvelle-Calédonie et en Polynésie française, ont fait l'objet de com- munications lors de symposiums $(16,17)$. Une méthodologie pour apprécier la sensibilité des sols à l'érosion et l'érosion elle-même a été proposée (16). La nécessité s'impose pour tout aire marine protégée de voir établie une réglementation environnementale des bassins versants dans une approche avec les communautés villageoises locales (17). Il est certain que ces recherches donneront lieu ultérieurement à des publications. Elles contribuent à renforcer l'indispensable nécessité d'une gestion intégrée des zones côtières tropicales, du sommet de la montagne au bord du récif extérieur, selon d'ailleurs la conception environnementale traditionnelle des populations.

Les actions du CRISP sur la restauration des récifs coralliens dégradés ont été de transmettre le savoir-faire des techniques acquises par des équipes françaises aux pays cibles du CRISP lors d'opérations-terrain dès la fin 2005 avec l'installation et le suivi de deux sites pilotes aux Fiji et à Tuvalu. Un autre site a été identifié ultérieurement aux Samoa occidentales. Dans chacun des sites, les procédures étalées dans le temps sont les mêmes : réunions avec les communautés riveraines et les partenaires interagissant sur les récifs, formulation d'un projet de restauration, récolte de coraux sur un site donneur, transport et transplantation des coraux dans les sites à restaurer, suivi du chantier. L'ensemble des opérations se conduit avec une formation technique des communautés ayant adopté le projet pour duplication éventuelle ultérieure. Deux sites ont donc été restaurés et un troisième site de restauration aux Samoa occidentales est en cours d'étude. Ces opérations de restauration sont bien engagées et le savoir-faire passé dans les populations riveraines en même temps que les résultats incitent le secteur public comme le secteur privé, en particulier touristique, à développer de telles actions. Des publications font état de ces travaux, appliqués certes et d'intérêt local, mais qui renforcent les connaissances et pratiques en ce domaine dans le contexte mondial (symposiums 2, 4, 15 ; rapport 16 Fiji, rapport 13 Tuvalu)

La co-édition d'un manuel sur la restauration récifale en anglais est dès à présent à mettre à l'actif du CRISP (rapport 4). Initialement prévu en fin de programme, ce projet a connu un développement imprévu à travers la concrétisation d'un partenariat avec le groupe de travail « Restauration et remédiation ", université de Newcastle, rattaché au programme Coral Reef Targeted Research and Capacity Building for Management, financé par le GEF et la Banque mondiale (projet d'envergure mondiale qui vise le développement de pôles d'excellence à travers 
la planète dans le domaine de la recherche scientifique en milieu récifal). La confrontation des savoir-faire techniques entre ce groupe de travail et l'action "restauration » du CRISP a permis leur association pour l'élaboration d'un guide Reef restoration concepts and guidelines : making sensible management choices in the face of uncertainty (rapport). Le guide sera prochainement édité en français sous l'égide du CRISP et de l'IFRECOR. L'édition d'un manuel plus complet est aussi prévue pour 2008-2009, dont la qualité et l'exhaustivité vont au-delà de ce que l'action CRISP restauration était à même de fournir à elle seule. Cette production complétera les ouvrages déjà consacrés à la restauration des récifs coralliens

Notons enfin des travaux d'intérêt régional sur la gestion des habitats et ressources récifolagonaires, sur les politiques publiques et la gouvernance (symposiums 6, 7,8). Ces travaux alimentent la réflexion générale mondiale sur ces thèmes.

Axe 3: Les progrès de la connaissance sur de nouvelles ressources et leur potentiel d'exploitation économique

Dans ce domaine, les recherches ont concerné trois thèmes dans le programme CRISP: la collecte et l'exploitation des larves de poissons, les substances marines actives au plan pharmacodynamique et l'exploitation des coraux en aquariophilie.

Les larves de poissons comme nouvelle ressource d'intérêt économique nécessitent l'exposé du contexte « découverte scientifique, perspectives d'application, projets de développement » : la presque totalité des organismes vivant dans les récifs coralliens se reproduisent par émissions en pleine eau de produits génitaux mâles et femelles qui donnent des larves. Celles-ci sont dispersées dans l'océan, pendant des durées allant de quelques semaines à quelques mois selon les espèces, avant de se retrouver à proximité de récifs coralliens où, sous forme de post-larves, elles vont investir les habitats pour rejoindre les populations adultes de même espèce. C'est ce que l'on nomme le «recrutement». Les post-larves reviennent dans les récifs coralliens selon un calendrier lunaire précis et des recherches dans les années 1990 ont montré qu'elles passaient en quantité considérable le front récifal pour pénétrer dans les lagons. Des filets pour les collecter ont été mis au point. Ces milliards de post-larves vont être presque toutes victimes des prédateurs. L'idée de les sauver et de les récupérer pour les élever en aquarium trouve son intérêt lorsqu'il s'agit d'espèces commerciales. C'est l'aventure qui a été tentée après cette découverte. La récupération des post-larves et leur élevage intéressent les espèces du marché de l'aquariophilie et trouvent aussi une valorisation dans les essais de réensemencement en larves de récifs dégradés ou surexploités. En Polynésie française, d'où est originaire cette découverte et ses perspectives, le territoire et des entreprises travaillent dans ces directions. L'objectif de l'action "capture et valorisation des larves de poissons et de crustacés de récifs » du CRISP comprend un volet polynésien et un volet fidjien plus conséquent. Le volet polynésien a normalement suivi son cours et a débouché sur l'élaboration d'un Guide d'identification des larves de poissons récifaux de Polynésie française (rapport 3) en collaboration avec le service des Pêches de Polynésie française. Ce manuel constitue un outil précieux à la disposition des professionnels désirant se lancer dans la valorisation économique des post-larves. Cet ouvrage original de 104 pages, une avancée de la connaissance scientifique avec perspectives de développement d'activités alternatives pour l'économie des pays insulaires du Pacifique, comporte des centaines de photos de post-larves et d'adultes de plus de 140 espèces. L'action doit se développer par la réalisation en 2008 d'un guide des post-larves encore plus complet et en anglais à destination des pays anglophones.

Les opérations sur le terrain à Fiji tournent à plein régime depuis juillet 2006 avec le fonctionnement d'une ferme aquacole dans les locaux de l'usp au sein de laquelle un étudiant français forme des homologues fidjiens à la capture et l'élevage des post-larves, tout en réalisant une thèse sur l'optimisation des techniques de réensemencement en milieu naturel. Parallèlement, deux sites villageois fidjiens ont été identifiés où se réalise le transfert de technologie et le début d'opérations de terrain, les personnels sont encadrés techniquement et sur le plan socioéconomique, afin de débuter une filière postlarves aux Fiji. Ce travail ne s'accomplit pas sans difficultés que soulignent les partenaires : choix de sites présentant des problèmes (pollution et faible recrutement larvaires), épizooties, difficultés à intéresser les pêcheurs locaux.

Les publications scientifiques relatives à la découverte des conditions du recrutement des larves de poissons dans les récifs coralliens, dans les années 1990, ont été antérieures au début du programme CRISP. Toutefois des synthèses sur ce sujet et sur les perspectives et réalisations appliquées à ce sujet on fait l'objet de communication lors de symposiums $(25,26,27,28)$ et d'article en préparation (article 14 pour Fish Information 
Bulletin). Les méthodes de collecte de ces larves recrutantes ont été exposées (article 12). Il en est de même des résultats des expérimentations menées sur le terrain aux Fiji (symposium 22). L'important document qui diffuse cette connaissance et ses ouvertures vers l'exploitation de cette nouvelle ressource est un rapport de 76 pages largement illustré et documenté (rapport 1). Dans une perspective exploratoire, des résultats ont été établis sur les conditions d'élevage multi espèces des larves en aquarium (rapport 12). Les problèmes du marché des poissons d'aquarium ont été abordés dans un article afin de promouvoir une filière économique sur l'exploitation de cette ressource dans le Pacifique Sud (article 13 dans Fisheries Science).

Ce qui vient d'être exposé sur les larves de poissons est tout aussi vrai sur les larves des milliers d'espèces d'invertébrés vivant dans les récifs. Mais les études à leur sujet ne font que débuter car la reconnaissance des larves qui recrutent (mollusques, crustacés, échinodermes pour citer des espèces pouvant être d'intérêt commercial, mais aussi les coraux) est encore impossible au niveau spécifique, sauf exception. Néanmoins, cette voie de recherche pour la décennie à venir est déjà dans les préoccupations des scientifiques et des gestionnaires. Signalons enfin une communication sur le commerce des coraux en aquariophilie (symposium 29) qui devient préoccupante parce qu'elle repose dans le Pacifique Sud sur des collectes de jeunes individus ou de boutures dans le milieu naturel.

Ces recherches appliquées qui amènent un progrès des connaissances fondamentales sur les cycles de vie des poissons coralliens, sont valorisées par le programme CRISP. Le bien fondé de cette orientation a été ressenti par l'AFD et le FFEM qui ont décidé en 2007 de crédits complémentaires au programme CRISP. L'objectif étant d'aller plus avant dans ce domaine qui permet d'élargir la gamme des activités génératrices de revenu pour les populations riveraines et, notamment, celles à proximité d'aires marines protégées qui doivent bien souvent, au moins sur le court terme, renoncer à tout ou partie des revenus traditionnels tirés de la pêche ou de la collecte.

La recherche de substances actives au plan pharmacodynamique constituait une des actions de CRISP. Elle s'est révélée féconde en se focalisant en un premier temps sur la collecte d'organismes des îles Salomon. Un très grand nombre d'extraits d'organismes récifaux (éponges et algues) se sont révélés intéressants dont les principes actifs sont en cours d'étude : (article 11, symposiums $30,31,32,33,34)$ pour la plupart des communications présentées à l'European conference on marine natural products. Les substances actives sont bien plus nombreuses que prévues. Cette recherche est soutenue par l'intérêt que portent les Salomon à l'éventuelle découverte de molécules antipaludiques compte tenu du fléau que représente le paludisme dans ces îles. Ces recherches s'accompagnent d'un volet juridique visant à améliorer les législations des pays insulaires pour le partage des bénéfices issus de la valorisation des substances actives marines (rapports 8, 9).

\section{Axe 4 : les progrès dans la maîtrise de nouveaux outils de surveillance de l'état des habitats et des ressources et de leur gestion}

Dans ce domaine, les résultats des actions CRISP seront évoqués selon plusieurs rubriques : techniques d'observation et de relevés sur le terrain, pêcheries et indicateurs, aspects socioéconomiques, gestion et gouvernance

De nouvelles méthodes de prospection sur le terrain ont été mises au point pour la surveillance de l'état des récifs à des échelles spatiales assez larges. Elles reposent sur l'usage de caméras sous-marines avec des protocoles particulièrement adaptés aux comptages de poissons (article 15, symposiums 36, 40, 41, 48, 49, 53, 54). Ces nouvelles techniques d'observations et de comptages permettent de diminuer considérablement le temps de travail sur le terrain au profit, pour un même résultat, d'un plus important investissement dans le temps de dépouillement des données et de leur traitement.

L'estimation de la pêche de plaisance (récréationnelle) a été abordée en Nouvelle-Calédonie et mérite d'être signalée compte tenu de la rareté de telles études dans le Pacifique sud. (symposium 43). La recherche d'indicateurs sur les pêcheries est toujours un sujet d'actualité auquel la communication lors du symposium 39 a contribué avec une analyse critique de divers indicateurs.

Les aspects socio-économiques sont de plus en plus pris en compte pour les populations riveraines des récifs coralliens, particulièrement dans le cadre d'aires marines protégées qui sont mises en place et qui exigent une contrepartie pour les populations eu égard à la pêche interdite ou réglementée. Les communications lors de symposiums et les rapports sont les préludes à des publications scientifiques qui sortiront prochainement (symposiums 50, 51, 52 ; rapports 7,10 ).

Enfin les problèmes de gestion des habitats et des ressources ont été évoqués dans de nombreuses communications lors de symposiums. Elles sont toutes centrées sur l'importance de la parti- 
cipation des communautés riveraines et sur les difficultés de gestion lorsque des réglementations de protection sont mises en place (symposiums $35,37,38,45,46,47,55,56$; rapport 20 ). Elles traitent parfois de la perception, sous différents aspects, de la biodiversité des populations (ethnobiodiversité) et de son importance pour toute compréhension et adhésion des populations à des mesures de préservation de la ressource (symposiums 42, 44).

\section{Les moyens de diffusions des connaissances}

La diffusion des connaissances scientifiques acquises au profit de la communauté scientifique s'est faite par des articles dans des périodiques scientifiques et par des communications lors de congrès et symposiums. Si nous avons analysé les rapports (rapports à diffusion restreinte, qualifiés de littérature grise, mais toutefois consultable sur le site du CRISP), c'est en raison du potentiel de publications qu'offrent pour l'avenir les résultats qui y figurent.

Nous noterons que des articles (15) ont été publiés ou sont sous presse ou en instance de dépôt dans les périodiques suivants : Science, Journal of Experimental Marine Biology and Ecology, Coral Reefs, Aquatic Biology, Oecologia, Phycological science, Pacific science, Talanta, Contribution University of Michigan, Organic letters (American Chemical Society), Fisheries science, Fish information bulletin, SPC Fisheries newletter, SPC Life reef fish information bulletin.

Les congrès, colloques et symposiums au cours desquels des résultats scientifiques du programme CRISP ont été exposés (56), sont divers :

- GECOREv, Saint-Quentin-en-Yvelines, juin 2006 ;

- Third international symposium on stock enhancement and sea ranching, Seattle, septembre 2006 ;
- International Tropical Marine Ecosystem Management Symposium, Mexico, octobre 2006 ;

- BIODEC, Nouméa, décembre 2006 ;

- Pacific Science Congress, PSA Pacific Science Association, Okinawa, juin 2007 ;

- Asia Pacific Coral Reef Symposium, Hong Kong, juin 2007 ;

- Forum on the sustainable development of Pacific coastal resources, Townsville, septembre 2007 ;

- European Marine Protected Area Symposium, Murcia, septembre 2007 ;

- European conference on marine natural products, Naples, septembre 2007 ;

- $6^{\text {th }}$ European conference on ecological modelling, Trieste, novembre 2007 ;

- Pacific island countries GIS/RS user conference, Suva, décembre 2007 ;

- International Coral Reef Symposium, ICRs, Floride, juillet 2008.

On notera que les partenaires du programme CRISP ont réellement fait des efforts de communication lors de trois symposiums :

- International Coral Reef Symposium, ICRS, Floride, juillet 2008 avec quinze communications sans compter quelques autres non encore recensées au moment de la rédaction de ce rapport;

- Pacific Science Congress, PSA Pacific Science Association, Okinawa, juin 2007 avec quinze communications ;

- European Marine Protected Area Symposium, Murcia, septembre 2007 avec sept communications

$\mathrm{Au}$ total, nous avons donc considéré dans ce travail quinze publications scientifiques, cinquante-six communications à congrès et vingt-cinq rapports dont les listes se trouvent ci-dessous en annexe. 


\section{Annexes}

\section{A. Articles dans des périodiques scientifiques}

\begin{tabular}{|c|c|c|c|c|c|c|}
\hline Axe & $\mathbf{N}^{\circ}$ & Auteurs & Année & Article & Périodique & Nb pages \\
\hline \multirow[t]{5}{*}{1} & 1 & Poupin & 2007 & Zoogeography of the crustacea in the tropical Pacific: & Pacific Science & sous presse \\
\hline & 2 & $\begin{array}{l}\text { N'Yeurt, Wynne, } \\
\text { Payri }\end{array}$ & 2007 & $\begin{array}{l}\text { Myriogramme melanesiensis and } M \text {. heterostroma } \\
\text { (Delesseriaceae, Rhodophyta), two new species from the } \\
\text { Solomon islands and Vanuatu }\end{array}$ & $\begin{array}{l}\text { Contribution University of Michigan } \\
\text { Herbarium } 25\end{array}$ & $\begin{array}{l}12 \text { pages } \\
\text { pp. } 213-224\end{array}$ \\
\hline & 3 & N'Yeurt, Payri & 2007 & $\begin{array}{l}\text { Grammephora peyssonnelioides gen. et } s p . n o v . \\
\text { (Rhodophyta, Rhodymeniaceae) from the Solomon Islands, } \\
\text { South Pacific }\end{array}$ & Phycological research 55 & $\begin{array}{l}9 \text { pages } \\
\text { pp. } 286-294\end{array}$ \\
\hline & 4 & \begin{tabular}{|l|} 
Le Lann, \\
Kervarec, Payri, \\
Deslandes, Stiger \\
\end{tabular} & 2007 & $\begin{array}{l}\text { Discrimination of allied species within the genusTurbinaria } \\
\text { (Fucales, Phaeophyceae) using HRMASNMR spectroscopy }\end{array}$ & $\begin{array}{l}\text { Talanta, in press (Available online at } \\
\text { www.sciencedirect.com) }\end{array}$ & 5 pages \\
\hline & 5 & Beurier & 2007 & La protection juridique de la biodiversité marine & $\begin{array}{l}\text { Dalloz - collection Pour un droit commun } \\
\text { de l'environnement : mélanges en } \\
\text { l'honneur de Michel Prieur }\end{array}$ & pp. $803-815$ \\
\hline \multirow[t]{5}{*}{2} & 6 & $\begin{array}{l}\text { Lecchini, Planes, } \\
\text { Galzin }\end{array}$ & 2006 & $\begin{array}{l}\text { The influence of habitat characteristics and conspecifics on } \\
\text { attraction and survival of coral reef fish juveniles }\end{array}$ & $\begin{array}{l}\text { Journal of Experimental Marine Biology } \\
\text { and Ecology }\end{array}$ & 6 pages \\
\hline & 7 & $\begin{array}{l}\text { Lecchini, } \\
\text { Osenberg, } \\
\text { Shima, } \\
\text { St Mary. Galzin } \\
\end{array}$ & 2007 & $\begin{array}{l}\text { Ontogenetic changes in habitat selection during settlement } \\
\text { in a coral reef fish: ecological determinants and sensory } \\
\text { mechanisms }\end{array}$ & Coral Reefs 26 & $\begin{array}{l}10 \text { pages } \\
\text { pp. } 423-432\end{array}$ \\
\hline & 8 & $\begin{array}{l}\text { Glenn, Almany,. } \\
\text { Berumen, } \\
\text { Thorrold, Planes, } \\
\text { Jones. }\end{array}$ & 2007 & $\begin{array}{l}\text { Local Replenishment of Coral Reef Fish Populations in a } \\
\text { Marine Reserve }\end{array}$ & Science & 4 pages \\
\hline & 9 & $\begin{array}{l}\text { Mellin, Grüss, } \\
\text { Ponton }\end{array}$ & en prep. & $\begin{array}{l}\text { Spatial patterns in abundance and } \\
\text { growth of a juvenile coral fish: covariation with } \\
\text { miucrohabitat characteristics }\end{array}$ & Aquatic Biology & en préparation \\
\hline & 10 & \begin{tabular}{|l|} 
Mellin, Galzin, \\
Ponton, Vigliola
\end{tabular} & en prep. & $\begin{array}{l}\text { Back-calculated larval and juvenile growth trajectories of } \\
\text { coral reef fish: How to untangle fast growth and } \\
\text { selection for fast growth }\end{array}$ & Oecologia & en préparation \\
\hline \multirow[t]{4}{*}{3} & 11 & & 2007 & $\begin{array}{l}\text { Debromodispacamides B and D: Isolation from the Marine } \\
\text { Sponge Agelas mauritiana and Stereoselective Synthesis }\end{array}$ & $\begin{array}{l}\text { Organic letters (American Chemical } \\
\text { Society) 0, 0 A-D DOI: }\end{array}$ & 4 pages \\
\hline & 12 & Lecaillon, Lourie & 2007 & $\begin{array}{l}\text { Current status of marine post-larvae collection: Existing } \\
\text { tools, initial results, market opportunities and prospects }\end{array}$ & Life reef fish information bulletin (CPS) & pp. 3-10 \\
\hline & 13 & $\begin{array}{l}\text { Lecchini, Polti, } \\
\text { Nakamura } \\
\end{array}$ & 2007 & $\begin{array}{l}\text { New perspectives on aquarium fish } \\
\text { trade }\end{array}$ & Fisheries science 72 & $\begin{array}{ll}8 \text { pages } \quad p p \\
40-47\end{array}$ \\
\hline & 14 & $\begin{array}{l}\text { Malpot, Galzin, } \\
\text { Remoissenet }\end{array}$ & en prep. & $\begin{array}{l}\text { Utilisation des mlarves de poissons } \\
\text { des récifs coralliens: synthèse des }\end{array}$ & Fish Information Bulletin & en préparation \\
\hline 4 & 15 & \begin{tabular}{|l|} 
Langlois, \\
Chabanet, \\
Pelletier, Harvey
\end{tabular} & 2006 & $\begin{array}{l}\text { Baited underwater video for assessing reef fish populations } \\
\text { in marine reserves }\end{array}$ & SPC Fisheries Newsletter 118 & 5 pages \\
\hline
\end{tabular}

\section{B. Communications dans des symposiums, colloques et congrès}

\begin{tabular}{|c|c|c|c|c|c|c|}
\hline Axe & $n^{\mathbf{o}}$ & Auteurs & Communication & Symposium & Lieu & Date \\
\hline 1 & 1 & De Mazières & $\begin{array}{l}\text { Habitat mapping of the } \\
\text { Coral Coast reefs, Fiji } \\
\text { Islands }\end{array}$ & $\begin{array}{l}\text { Pacific Island } \\
\text { Countries GIS/RS } \\
\text { User Conference }\end{array}$ & Fiji/Suva & $\begin{array}{l}4-7 \text { déc. } \\
2007\end{array}$ \\
\hline \multirow[t]{3}{*}{2} & 2 & Job & $\begin{array}{l}\text { SPI INFRA/FSPI, Case } \\
\text { study 1: Coral displace- } \\
\text { ment and reef restora- } \\
\text { tion as a compensatory } \\
\text { measure to a prot cons- } \\
\text { truction, New Caledo- } \\
\text { nia ; Case study 2: Reef } \\
\text { restoration through low } \\
\text { cost methods and invol- } \\
\text { vement of local com- } \\
\text { munities, Fiji Island } \\
\end{array}$ & $\begin{array}{l}\text { International } \\
\text { Tropical Marine } \\
\text { Ecosystem } \\
\text { Management } \\
\text { Symposium } \\
\text { (ITMEM) }\end{array}$ & $\begin{array}{l}\text { Meximo/ } \\
\text { Cozumel }\end{array}$ & $\begin{array}{l}16-20 \text { oct- } \\
2006\end{array}$ \\
\hline & 3 & Salvat & $\begin{array}{l}\text { Coral reef monitoring } \\
\text { in French Polynesia: } \\
\text { objectives, manage- } \\
\text { ment and results }\end{array}$ & \multirow[t]{2}{*}{$\begin{array}{l}\text { Pacific Science } \\
\text { Congress } 21^{\text {th }}\end{array}$} & \multirow[t]{2}{*}{$\begin{array}{l}\text { Japon/ } \\
\text { Okinawa }\end{array}$} & \multirow[t]{2}{*}{ Juin 2007} \\
\hline & 4 & $\begin{array}{l}\text { Egretaud, Jouvin, Guille- } \\
\text { met, Boudet, Mathieu }\end{array}$ & $\begin{array}{l}\text { Rehabilitation of a } \\
\text { lagoonary area under } \\
\text { Stegastes colonisation }\end{array}$ & & & \\
\hline
\end{tabular}




\begin{tabular}{|c|c|c|c|c|c|}
\hline 5 & $\begin{array}{l}\text { Lecchini, Dumas, Mills, } \\
\text { Banaigs, Ponton }\end{array}$ & $\begin{array}{l}\text { Effects of alternate } \\
\text { coral reef states on the } \\
\text { attraction, settlement } \\
\text { and subsequent } \\
\text { survival of marine } \\
\text { invertebrates } \\
\text { and fish larvae }\end{array}$ & \multirow[t]{4}{*}{$\begin{array}{l}\text { Pacific Science } \\
\text { Congress } 21^{\text {th }}\end{array}$} & \multirow[t]{4}{*}{$\begin{array}{l}\text { Japon/ } \\
\text { Okinawa }\end{array}$} & \multirow[t]{4}{*}{ Juin 2007} \\
\hline 6 & Brenier, Ferraris, Galzin & $\begin{array}{l}\text { Community based } \\
\text { monitoring of coral } \\
\text { reef fish resources in } \\
\text { two contrasted islands } \\
\text { of French Polynesia }\end{array}$ & & & \\
\hline 7 & Clua, Legendre & $\begin{array}{l}\text { Potential indicators } \\
\text { of the status of reef } \\
\text { fisheries based on the } \\
\text { shifting dominance } \\
\text { phenomenon among } \\
\text { scarid species } \\
\text { submitted to fishing } \\
\text { pressure }\end{array}$ & & & \\
\hline 8 & $\begin{array}{l}\text { Herrenschmidt, Mangeas, } \\
\text { Dumas, Despinoy, David }\end{array}$ & $\begin{array}{l}\text { Some hints about the } \\
\text { impacts of watershed } \\
\text { management on MPAS } \\
\text { within the South Pacific } \\
\text { area }\end{array}$ & & & \\
\hline 9 & & $\begin{array}{l}\text { Natural and } \\
\text { anthropogenic } \\
\text { influences on the } \\
\text { diversity structure of } \\
\text { reef fish communities } \\
\text { in the Tuamotu } \\
\text { Archipelago (French } \\
\text { Polynesia) }\end{array}$ & $\begin{array}{l}6^{\text {th }} \text { European } \\
\text { Conf. On Ecolo- } \\
\text { gical Modelling }\end{array}$ & Trieste, Italie & $\begin{array}{l}\text { Novembre } \\
2007\end{array}$ \\
\hline 10 & $\begin{array}{l}\text { Mills, Dumas; Lecchini, } \\
\text { Parmentier }\end{array}$ & $\begin{array}{l}\text { Recruitment potential } \\
\text { and recruitment } \\
\text { survival as a function } \\
\text { of habitat degradation }\end{array}$ & \multirow[t]{5}{*}{$\begin{array}{l}\text { ICRS Int. Coral } \\
\text { Reef } \\
\text { Symposium }\end{array}$} & \multirow[t]{5}{*}{ Floride, USA } & \multirow[t]{5}{*}{\begin{tabular}{|l} 
Juillet \\
2008
\end{tabular}} \\
\hline 11 & Mellin, Gruss, Ponton & $\begin{array}{l}\text { Special patterns in } \\
\text { abundances and growth } \\
\text { of a juvenile coral reef } \\
\text { fish: covariation with } \\
\text { microhabitats } \\
\text { characteristics }\end{array}$ & & & \\
\hline 12 & Lowell, Sykes & $\begin{array}{l}\text { Rapid recovery from } \\
\text { bleaching events - Fiji } \\
\text { global coral reef } \\
\text { monitoring network } \\
\text { assessment of hard } \\
\text { coral cover from } \\
\text { 1999-2007 }\end{array}$ & & & \\
\hline 13 & $\begin{array}{l}\text { Lowell, Sykes, Bonito, } \\
\text { Khan, Ponton }\end{array}$ & $\begin{array}{l}\text { Monitoring seawater } \\
\text { temperature and coral } \\
\text { community response } \\
\text { across Fiji - an } \\
\text { archipelago-wide } \\
\text { monitoring programme }\end{array}$ & & & \\
\hline 14 & $\begin{array}{l}\text { Lecchini, Dumasi, Mills, } \\
\text { Parmentier Banaigs, Pon- } \\
\text { ton }\end{array}$ & $\begin{array}{l}\text { Effects of alternate } \\
\text { coral reef states on the } \\
\text { attraction, settlement } \\
\text { and subsequent } \\
\text { survival of marine } \\
\text { invertebrates and fish } \\
\text { larvae }\end{array}$ & & & \\
\hline
\end{tabular}




\begin{tabular}{|c|c|c|c|c|c|c|}
\hline & 15 & Job & $\begin{array}{l}\text { Lessons learnt from } \\
\text { applied restoration } \\
\text { projects }\end{array}$ & $\begin{array}{l}\text { ICRS Int. Coral } \\
\text { Reef } \\
\text { Symposium }\end{array}$ & Floride, USA & \begin{tabular}{|l|} 
Juillet \\
2008
\end{tabular} \\
\hline & 16 & Dumas, Printemps, Fossey & $\begin{array}{l}\text { The risk assessment of } \\
\text { soil erosion for better } \\
\text { management of coral } \\
\text { reefs in the Pacific }\end{array}$ & & & \\
\hline & 17 & $\begin{array}{l}\text { David, Dumas, Despinoy, } \\
\text { Mangeas, Herrenschmidt }\end{array}$ & $\begin{array}{l}\text { Some hints about the } \\
\text { impacts of watershed } \\
\text { management on MPAS } \\
\text { in the South Pacific } \\
\text { Islands }\end{array}$ & & & \\
\hline & 18 & Planes et al. & $\begin{array}{l}\text { Genetic parental } \\
\text { analysis reveals both } \\
\text { local retention and } \\
\text { large scale connectivity } \\
\text { of clown fish in Kimbe } \\
\text { Bay }\end{array}$ & & & \\
\hline & 19 & Planes et al. & $\begin{array}{l}\text { Absence of geographic } \\
\text { barrier across the } \\
\text { indo-pacific province } \\
\text { for coral reef fishes }\end{array}$ & & & \\
\hline & 20 & $\begin{array}{l}\text { Salvat, Chancerelle, Lison } \\
\text { de Loma, Penin, Sievenart, } \\
\text { Lecouvreur, Aubanel, } \\
\text { Monier, lagouy, Asjeroud }\end{array}$ & $\begin{array}{l}\text { Evidence of an } \\
\text { outbreak of } \\
\text { crown-of-thorns } \\
\text { starfish (COTS) in } \\
\text { French Polynesia: } \\
\text { observations between } \\
2005 \text { and } 2007\end{array}$ & & & \\
\hline & 21 & $\begin{array}{l}\text { Adjeroud, Michonneau, } \\
\text { Edmunds, Chancerelle, } \\
\text { Penin, Vidal-Dupiol, } \\
\text { Salvat, Galzin }\end{array}$ & $\begin{array}{l}\text { Recurrent large-scale } \\
\text { disturbances, recovery } \\
\text { trajectories and } \\
\text { resilience of coral } \\
\text { assemblages on a coral } \\
\text { reef in the South } \\
\text { Central Pacific }\end{array}$ & & & \\
\hline & 22 & MacKay, Morris & $\begin{array}{l}\text { Coral reef Monitorings } \\
\text { in the South West } \\
\text { Pacific }\end{array}$ & & & \\
\hline & 23 & $\begin{array}{l}\text { Kulbicki, Vigliola, } \\
\text { Wantiez, Galzin } \\
\text { MouTham }\end{array}$ & $\begin{array}{l}\text { Life-history traits of } \\
\text { rare coral reef fishes of } \\
\text { the South Pacific region }\end{array}$ & & & \\
\hline & 24 & $\begin{array}{l}\text { Kulbicki, Vigliola, } \\
\text { Wantiez, MouTham }\end{array}$ & $\begin{array}{l}\text { The role of functionnal } \\
\text { in diversity-biomass } \\
\text { relationships, case } \\
\text { study of reef fishes }\end{array}$ & & & \\
\hline 3 & 25 & Lourié, Gilles Lecaillon & $\begin{array}{l}\text { Post-larval collection } \\
\text { and rearing for } \\
\text { restocking of marine } \\
\text { protected areas }\end{array}$ & $\begin{array}{l}\text { Third Internatio- } \\
\text { nal Symposium } \\
\text { on Stock Enhan- } \\
\text { cement and Sea } \\
\text { ranching }\end{array}$ & $\begin{array}{l}\text { Canada/ } \\
\text { Seattle }\end{array}$ & $\begin{array}{l}\text { Sep- } \\
\text { tembre } \\
2006\end{array}$ \\
\hline & 26 & $\begin{array}{l}\text { Galzin, Grignon, } \\
\text { Remoissenet }\end{array}$ & $\begin{array}{l}\text { More than one decade } \\
\text { of fish larvae fishing in } \\
\text { French Polynesia }\end{array}$ & $\begin{array}{l}\text { Pacific Science } \\
\text { Congress } 21^{\text {th }}\end{array}$ & \begin{tabular}{|l} 
Japon/ \\
Okinawa
\end{tabular} & Juin 2007 \\
\hline & 27 & Kirata, Vieux, Clua & $\begin{array}{l}\text { PCC (Post Larval } \\
\text { Capture and Culture) } \\
\text { potential afetr one year } \\
\text { of collection in Fiji } \\
\text { within -the CRISP } \\
\text { programme }\end{array}$ & & & \\
\hline
\end{tabular}




\begin{tabular}{|c|c|c|c|c|c|c|}
\hline & 29 & $\begin{array}{l}\text { Galzin, R., Lecaillon, G., } \\
\text { Lipchitz, A., Malpot, E., } \\
\text { Viller, P. }\end{array}$ & $\begin{array}{l}\text { Three experiences of } \\
\text { post larval capture and } \\
\text { culture (PCC) in the } \\
\text { Indo-Pacific: technical, } \\
\text { ecological and } \\
\text { socio-economical } \\
\text { observations } \\
\text { Live Rock and Live } \\
\text { Coral Trade in Fiji, } \\
\text { Culture vs. Wild } \\
\text { harvest, financial } \\
\text { assessment, issues and } \\
\text { ways forward. }\end{array}$ & $\begin{array}{l}\text { Forum on the } \\
\text { sustainable } \\
\text { development of } \\
\text { Pacific Coastal } \\
\text { Resources }\end{array}$ & $\begin{array}{l}\text { Australie/ } \\
\text { Townsville }\end{array}$ & $\begin{array}{l}3-7 \quad \text { sep } \\
\text { tembre } \\
2007\end{array}$ \\
\hline & 30 & $\begin{array}{l}\text { Appenzeller, Adeline, } \\
\text { Martin, Gallard, Boury- } \\
\text { Esnault, Petek, Valentin, } \\
\text { Zaparucha, Al-Mourabit, } \\
\text { Debitus }\end{array}$ & $\begin{array}{l}\text { Agelasines } \mathrm{J} \text { and } \mathrm{K} \\
\text { from the Solomon } \\
\text { Islands Marine Sponge } \\
\text { Agelas sp./Poster }\end{array}$ & \multirow[t]{5}{*}{$\begin{array}{l}\text { European confer- } \\
\text { ence on marine } \\
\text { natural products }\end{array}$} & \multirow[t]{5}{*}{ Naples/Italie } & \multirow[t]{5}{*}{$\begin{array}{l}\text { 16-21 sep } \\
\text { tembre } \\
2007\end{array}$} \\
\hline & 31 & $\begin{array}{l}\text { J. Appenzeller, C. Debitus, } \\
\text { M.T. Martin, } \\
\text { M.T. Adeline, J.F. Gallard, } \\
\text { E. Tran Huu Dau, } \\
\text { A. Zaparucha, } \\
\text { A. Al-Mourabit }\end{array}$ & $\begin{array}{l}\text { Non brominated } \\
\text { pyrrole-2 } \\
\text { amino-imidazoles } \\
\text { alkaloids from the } \\
\text { Pacific marine sponge } \\
\text { Agelas sp/Poster }\end{array}$ & & & \\
\hline & 32 & $\begin{array}{l}\text { J. Appenzeller, } \\
\text { M.T. Martin, } \\
\text { M.T. Adeline, } \\
\text { J.F. Gallard, } \\
\text { N. Boury-Esnault, } \\
\text { J. Hooper, A. Zaparucha, } \\
\text { C. Debitus and } \\
\text { A.Al-Mourabit } \\
\end{array}$ & $\begin{array}{l}\text { New non brominated } \\
\text { pyrrole-2amino- } \\
\text { imidazoles alkaloids } \\
\text { from Agelas } \\
\text { Cf mauritiana Pacific } \\
\text { sponge (Conference } \\
\text { programme) }\end{array}$ & & & \\
\hline & 33 & \begin{tabular}{|l|} 
Le Lann, Bourguet- \\
Kondracki, Longeon, \\
Ioannou, Roussis, Payri, \\
Debitus, Stiger-Pouvreau
\end{tabular} & \begin{tabular}{|l} 
Potent \\
antiinflammatory \\
active compounds from \\
marine brown algae \\
(Sargassaceae, \\
Fucales)/Poster
\end{tabular} & & & \\
\hline & 34 & $\begin{array}{l}\text { N. Bontemps-Subielos, } \\
\text { A Simon-Levert, } \\
\text { S. Lopez-Legentil, } \\
\text { B. Banaigs }\end{array}$ & $\begin{array}{l}\text { Pyridoacridine } \\
\text { alkaloids within purple } \\
\text { morphs of Cystodytes } \\
\text { spp. (Ascidiacea: } \\
\text { Polycitoridae) / } \\
\text { Poster }\end{array}$ & & & \\
\hline \multirow[t]{3}{*}{4} & 35 & $\begin{array}{l}\text { Eric Clua et Jean-Brice } \\
\text { Herrenschmidt }\end{array}$ & $\begin{array}{l}\text { Vers une gestion plus } \\
\text { « culturelle » des } \\
\text { milieux naturels en } \\
\text { Océanie }\end{array}$ & GECOREV & $\begin{array}{l}\text { France/ } \\
\text { Saint- } \\
\text { Quentin en } \\
\text { Yvelines }\end{array}$ & Juin 2006 \\
\hline & 36 & $\begin{array}{l}\text { Langlois, Anderson, } \\
\text { Cappo, Chabanet, Merritt, } \\
\text { Mouthan, Harvey, } \\
\text { Pelletier }\end{array}$ & $\begin{array}{l}\text { Baited remote } \\
\text { underwater video: a } \\
\text { tool for the assessment } \\
\text { and management of } \\
\text { coral reef fish/Forum } \\
\text { BIODEC ; } 30 \text { octobre - } \\
4 \text { novembre } 2006\end{array}$ & BIODEC & $\begin{array}{l}\text { Nouvelle- } \\
\text { Calédonie/ } \\
\text { Nouméa }\end{array}$ & $\begin{array}{l}30 \quad \text { octo- } \\
\text { bre }-4 \\
\text { novembre } \\
2006\end{array}$ \\
\hline & 37 & Ferraris & $\begin{array}{l}\text { Ecosystem approach } \\
\text { and indicators for } \\
\text { management of marine } \\
\text { resources and their uses } \\
\text { in Pacific islands }\end{array}$ & $\begin{array}{l}\text { CRISP Mooréa } \\
\text { workshop } \\
\text { France- } \\
\text { Japan-USA }\end{array}$ & $\begin{array}{l}\text { Polynésie } \\
\text { française/ } \\
\text { Mooréa }\end{array}$ & $\begin{array}{l}\text { Décembre } \\
2006\end{array}$ \\
\hline
\end{tabular}




\begin{tabular}{|c|c|c|c|c|c|}
\hline 38 & Léopold, Herrenschmidt & $\begin{array}{l}\text { Building the } \\
\text { co-management of } \\
\text { fisheries in customary } \\
\text { marine areas in Ouvéa, } \\
\text { New-Caledonia/ } \\
\text { Colloque international } \\
\text { BIODEC Novembre } 2006 \\
\text { (Nouméa) } \\
\end{array}$ & BIODEC & $\begin{array}{l}\text { Nouvelle- } \\
\text { Calédonie/ } \\
\text { Nouméa }\end{array}$ & $\begin{array}{l}\text { Décembre } \\
2006\end{array}$ \\
\hline 39 & $\begin{array}{l}\text { Ferraris, Léopold, Kro- } \\
\text { nen, Kulbicki, Labrosse }\end{array}$ & $\begin{array}{l}\text { Assessment of coral } \\
\text { reef fisheries by a } \\
\text { multidisciplinary panel } \\
\text { of indicators: a } \\
\text { comparative approach } \\
\text { of twelve sites in two } \\
\text { Pacific countries }\end{array}$ & \multirow[t]{7}{*}{$\begin{array}{l}\text { Pacific Science } \\
\text { Congress } 21^{\text {th }}\end{array}$} & \multirow[t]{7}{*}{$\begin{array}{l}\text { Japon/ } \\
\text { Okinawa }\end{array}$} & \multirow[t]{7}{*}{ Juin 2007} \\
\hline 40 & $\begin{array}{l}\text { Guillemot, Chabanet, Pel- } \\
\text { letier, Langlois, Leopold }\end{array}$ & $\begin{array}{l}\text { Impact of an expected } \\
\text { anthropogenic } \\
\text { disturbance in new } \\
\text { Caledonia: which } \\
\text { methods should be } \\
\text { used to monitor reef } \\
\text { fish communities? }\end{array}$ & & & \\
\hline 41 & MacKay, Morri, Sykes. & $\begin{array}{l}\text { Are we measuring the } \\
\text { Correct Parameters in } \\
\text { Coral Reef } \\
\text { Monitoring? Lessons } \\
\text { from the Southwest } \\
\text { Pacific node of the } \\
\text { Global Coral Reef } \\
\text { Monitoring Network }\end{array}$ & & & \\
\hline 42 & Randy Thaman & $\begin{array}{l}\text { Reef life and culture } \\
\text { under threat: The } \\
\text { conservation status of } \\
\text { copral reef biodiversity } \\
\text { and ethnobiodiversity } \\
\text { in the Pacific Islands }\end{array}$ & & & \\
\hline 43 & $\begin{array}{l}\text { Lollit-Boniface, Ferraris, } \\
\text { Lebigre, Pelletier, } \\
\text { Chabanet }\end{array}$ & $\begin{array}{l}\text { Recreational fishing } \\
\text { in New-Caledonie: } \\
\text { developing a social and } \\
\text { spatial analysis for } \\
\text { marine resource } \\
\text { management }\end{array}$ & & & \\
\hline 44 & Thaman & $\begin{array}{l}\text { The ecological } \\
\text { importance and } \\
\text { ethnobiodiversity of } \\
\text { Parrotfishes (Scaridae): } \\
\text { a pacific island } \\
\text { perspective }\end{array}$ & & & \\
\hline 45 & $\begin{array}{l}\text { Egretaud, Aubanel, Benet, } \\
\text { Jouvin, Monier, Verducci }\end{array}$ & $\begin{array}{l}\text { Marine zone manage- } \\
\text { ment plan (PGEM); a } \\
\text { modern tool fora } \\
\text { proactive management } \\
\text { plan of islander } \\
\text { communities }\end{array}$ & & & \\
\hline 46 & Chabanet, et al. & $\begin{array}{l}\text { From local knowledge } \\
\text { to management of a } \\
\text { coral reef ecosystem: } \\
\text { application to a mining } \\
\text { development project in } \\
\text { New Caledonia }\end{array}$ & $\begin{array}{l}\text { Asia Pacific } \\
\text { Coral Reef } \\
\text { Symposium }\end{array}$ & $\begin{array}{l}\text { Chine/Hong } \\
\text { Kong }\end{array}$ & Juin 2007 \\
\hline
\end{tabular}




\begin{tabular}{|c|c|c|c|c|c|}
\hline 47 & Clua & $\begin{array}{l}\text { Building Capacity and } \\
\text { Cooperation for } \\
\text { Sustainable } \\
\text { Development of Pacific } \\
\text { Coastal Resources } \\
\text { Forum Agenda final du } \\
\text { forum de Townsville } \\
\text { co-organisé par la } \\
\text { France et le CRISP }\end{array}$ & \begin{tabular}{|l} 
Townsville \\
Forum
\end{tabular} & $\begin{array}{l}\text { Australie/ } \\
\text { Townsville }\end{array}$ & $\begin{array}{l}3-7 \quad \text { sep- } \\
\text { tembre } \\
2007\end{array}$ \\
\hline 48 & $\begin{array}{l}\text { Emmanuelli, Ferraris, de } \\
\text { Loma, Osenberg \& Galzin. }\end{array}$ & $\begin{array}{l}\text { Assessing the initial } \\
\text { state of fish } \\
\text { assemblages to adapt } \\
\text { monitoring protocol } \\
\text { for managers in a } \\
\text { network of coral reef } \\
\text { Marine Protected } \\
\text { Areas: the example of } \\
\text { Moorea (French } \\
\text { Polynesia) }\end{array}$ & \multirow[t]{6}{*}{$\begin{array}{l}\text { European MPA } \\
\text { symposium }\end{array}$} & \multirow[t]{6}{*}{$\begin{array}{l}\text { Espagne/ } \\
\text { Murcia }\end{array}$} & \multirow[t]{6}{*}{$\begin{array}{l}25-28 \text { sep- } \\
\text { tembre } \\
2007\end{array}$} \\
\hline 49 & Emmanuelli, E.et al. & $\begin{array}{l}\text { Assessing the initial } \\
\text { state of fish } \\
\text { assemblages to adapt } \\
\text { monitoring protocol } \\
\text { for managers in a } \\
\text { network of MPAs/ } \\
\text { Poster }\end{array}$ & & & \\
\hline 50 & $\begin{array}{l}\text { Nicolas Pascal, Francesc } \\
\text { Maynou, Dominique } \\
\text { Pelletier \& Jocelyne } \\
\text { Ferrarris }\end{array}$ & $\begin{array}{l}\text { Contribution to the } \\
\text { design of new marine } \\
\text { protected areas } \\
\text { using the Isis-Fish } \\
\text { bioeconomic model: } \\
\text { the case of Solea } \\
\text { vulgaris and } \\
\text { Lithognathus } \\
\text { mormyrus fisheries in } \\
\text { Garraf (Spain, NW } \\
\text { Mediterranean) }\end{array}$ & & & \\
\hline 51 & $\begin{array}{l}\text { Nicolas Pascal, Nicolas } \\
\text { Maihota, Jocelyne Ferrar- } \\
\text { ris }\end{array}$ & $\begin{array}{l}\text { A bioeconomic } \\
\text { approach to assess the } \\
\text { implantation of small } \\
\text { size marine protected } \\
\text { areas and fishery } \\
\text { management strategies } \\
\text { using the ISIS Fish } \\
\text { model: the case of } \\
\text { Tikehau (French } \\
\text { Polynesia) commercial } \\
\text { fishery.. }\end{array}$ & & & \\
\hline 52 & $\begin{array}{l}\text { Pasal, N., Maynou, F., Pel- } \\
\text { letie, D., Mahevas, S., Fer- } \\
\text { raris, J. }\end{array}$ & $\begin{array}{l}\text { A bioeconomic } \\
\text { approach to assess the } \\
\text { design and impacts of } \\
\text { the creation of small } \\
\text { size MPA using the } \\
\text { ISIS-Fish model with } \\
\text { NPV approach }\end{array}$ & & & \\
\hline 53 & $\begin{array}{l}\text { Pelletier, Chabanet, Leleu, } \\
\text { Langlois, Hervé \& Guille- } \\
\text { mot. }\end{array}$ & $\begin{array}{l}\text { Video-based } \\
\text { observation techniques } \\
\text { for monitoring fish and } \\
\text { habitat in Marine } \\
\text { Protected Areas }\end{array}$ & & & \\
\hline
\end{tabular}




\begin{tabular}{|l|l|l|l|l|l|l|}
\hline 54 & $\begin{array}{l}\text { Pelletier, Leleu, } \\
\text { Mou-Tham, Chabanet, } \\
\text { Langlois, Hervé, } \\
\text { Guillemot }\end{array}$ & $\begin{array}{l}\text { Video-based } \\
\text { observation techniques } \\
\text { for monitoring fish } \\
\text { assemblages in coral } \\
\text { reef MPAs }\end{array}$ & $\begin{array}{l}\text { European MPA } \\
\text { symposiump }\end{array}$ & $\begin{array}{l}\text { Espagne/ } \\
\text { Murcia }\end{array}$ & $\begin{array}{l}\text { 25-28 sep- } \\
\text { tembre } \\
2007\end{array}$ \\
\hline 55 & $\begin{array}{l}\text { Herrenschmidy, Menu, } \\
\text { Hebert, Dumas }\end{array}$ & $\begin{array}{l}\text { Coral reef management } \\
\text { in the Pacific islands : } \\
\text { governance at risk }\end{array}$ & $\begin{array}{l}\text { 1icrs Int. Coral Symposium } \\
\text { Reef Sym }\end{array}$ & Floride, USA & $\begin{array}{l}\text { Juillet } \\
2008\end{array}$ \\
\hline 56 & Clua, Salvat & $\begin{array}{l}\text { Culture and updated } \\
\text { traditional } \\
\text { management tools for } \\
\text { serving ownership in } \\
\text { locally managed areas }\end{array}$ & & & \\
\hline
\end{tabular}

C. Rapports accessibles sur le site CRISP (www. crisponline.net)

1. Moana Initiative, 2007. Booklet on Post-larvae Capture and Culture of ( $P C C$ ) realized by the association Moana Initiative with a grant from Foundation Total. This document aims to raise political actors and sponsors awareness on the potential of those new trading channels in the field of sustainable fishing.

La PCC, un outil pour la conservation et la valorisation de la biodiversité, $76 \mathrm{p}$.

2. Juncker M., 2007. Jeunes poissons coralliens de Wallis et du Pacifique central, guide d'identification, Young coral reef fish of Wallis Islands and the Central Pacific, identification guide, livre du service territorial de l'Environnement de Wallis-etFutuna dans le cadre du programme CRISP, $170 \mathrm{p}$.

3. Maamaatuaiahutapu M., G. Remoissenet et R. Galzin, 2006. 2006 Guide d'identification des larves de poissons récifaux de Polynésie française. Éditions Talys. 104 p.

4. Edwards A.J. and E.D. Gomez, 2007. Reef Restoration Concepts and Guidelines: making sensible management choices in the face of uncertainty. Coral Reef Targeted Research \& Capacity Building for Management Programme: St Lucia, Australia. IV +38 pp.

5. Andrefouet, 2006. Atlas of Southeast Papua New Guinea Coral Reefs / Atlas Des Récifs Coralliens du Sud-Est de la Papouasie Nouvelle-Guinée.

6. Juncker Matthieu, 2006. A preliminary study of the risks and threats to the coastal ecosystems of New Caledonia.

7. O'Garra Tanya, 2007 (June). Final report: Estimating the total economic value (TEV) of the Navakavu LMma (Locally Managed Marine Area) in Vitu Levu island (Fiji).

8. 2007 (July). Analyse du cadre juridique lié à la valorisation des substances actives marines. Zone atelier $n^{\circ} 2$ - Salomon - Rapport préliminaire.

9. 2007 (July). Analyse du cadre juridique lié à la valorisation des substances actives marines. Zone atelier $n^{\circ} 1$ - Fiji-Rapport préliminaire.

10. David G., J.B. Herrenschmidt et E. Mirault, 2007 (juin). Valeur sociale et économique des récifs coralliens du Pacifique insulaire - Éléments méthodologiques

11. 2007 (juin). Panorama des méthodes d'analyse de l'érosion dans un contexte insulaire - Analyse méthodologique.

12. Vermond Sophie, 2007 (May). Development of multispecific postlarval fish rearing approach in aquarium. Internship report.

13. 2007 (June). Funafuti Atoll Coral Reef Restoration Project (Republic of Tuvalu) - Baseline Report.

14. Sykes Helen, 2007 (June). Status of coral reefs in the Fiji islands 2006.

15. 2007 (January). Reef Monitoring Rotuma Island (Fiji). Coral Reef Survey Report (November December 2006).

16. 2006 (December). Motoriki Restoration Site (Fiji) - Monitoring Report.

17. Lagouy Elodie, 2006. Reefcheck Polynesia - Activity Report 2006 / Reefcheck Polynésie - Rapport d'activités 2006.

18. Lagouy Elodie, 2007. Reefcheck Polynesia - Activity Report 2007 / Reefcheck Polynésie - Rapport d'activités 2007.

19. 2006 (6th-7th March). Global Coral Reef Monitoring Network (GCRMN) Fiji Review and Planning Meeting / wwF Coral Bleaching Rapid Response Communications Meeting.

20. Verducci Magali, . Faisabilité de la mise en place d'un plan de gestion des espaces maritimes (PGEM) à Alofi, Futuna-et-Wallis, Rapport de mission (3-17 mars 2007), Heremoana Consulting, Matthieu Juncker - Ibulu consultant en écologie marine.

21. Pichon Michel. Biodiversité des coraux Scléractiniaires de la Nouvelle-Calédonie. Phase 1. Rapport de mission (4-21 mai 2006).

22. Pichon Michel. Biodiversité des coraux Scléractiniaires de la Nouvelle-Calédonie. Phase 2. Rapport de mission (17 novembre-12 décembre 2006).

23. NeYeurt Antoine et Claude Payri, 2007 (mars). Liste des algues de l'expédition Santo 2006 (Vanuatu)

24. Lasne G., 2007 (March). Bibliographic synthesis on knowledge of coral reefs in New-Caledonia // Les coraux de la Nouvelle-Calédonie: synthèse bibliographique.

25. Poupin, 2007. Crustacés de Wallis-et-Futuna. 
